\title{
Double Descemet Membrane Forming a Double Anterior Chamber
}

\author{
Shmuel Graffi, Modi Naftali
}

\section{ABSTRACT}

Purpose: To report a case of double descemet membrane forming a double anterior chamber following 'Big bubble' DALK converted to PKP.

Results: A 52-year-old man with keratoconus underwent DALK operation converted to PKP due to macro perforation of the descemet membrane. On the postoperative evaluation a remnant of the host's descemet membrane formed a double anterior chamber. After a few months with no intervention, an attempt to deflect a thickened descemet membrane had failed, making entrance into the anterior chamber in order to excise the membrane inevitable. Two years following the second surgery a graft endothelial failure and cataract formation was documented, leading to a second corneal transplantation and lens implantation.

Conclusion: Double DM is an unusual condition following a DALK procedure which was converted into PKP. In the above case this complication resulted in multiple procedures and graft failure. We believe that early interventions such as gas injection or YAG laser could have resulted in a more favorable outcome.

Keywords: Double descemet membrane, Double anterior chamber, DALK, Big bubble.

How to cite this article: Graffi S, Naftali M. Double Descemet Membrane Forming a Double Anterior Chamber. J Kerat Ect Cor Dis 2013;2(3):129-132.

Source of support: Nil

Conflict of interest: None

\section{INTRODUCTION}

Deep anterior lamellar keratoplasty (DALK) using the big-bubble technique has recently become a widespread alternative to penetrating keratoplasty (PKP). ${ }^{1}$ One possible intraoperative complication is perforation of the host's descemet membrane (DM). While microperforations are usually left disregarded, macroperforations necessitate conversion into PKP and removal of the host's DM underlying the graft. ${ }^{2}$

In DALK, formation of a double anterior chamber is one possible immediate postoperative complication and caused by DM detachment from the donor's corneal stroma. ${ }^{3}$ Various treatment methods have included anterior chamber filling with air, perfluoropropane gas (C3F8), and sulfur hexafluoride gas (SF6). ${ }^{4}$ However, in some cases spontaneous resolution might occur. ${ }^{5}$ We report a case where the hosts DM was removed partially during DALK procedure that was converted into PKP causing a peculiar condition where double DM (host's and donor's) formed a double anterior chamber.

\section{CASE REPORT}

A 52-year-old man with keratoconus had preoperative visual acuities (VA) of 6/120 in the left eye (OS) and 6/10 in the right eye (OD). The patient underwent a big-bubble DALK procedure that was converted to PKP in the right eye. After performing a $7.50 \mathrm{~mm}$ diameter partial thickness trephination to a depth of 400 micron in the recipient cornea, a 30 gauge bent needle was introduced bevel down into the paracentral cornea, and a big bubble was formed. The superficial stroma was excised using an angle crescent knife. Penetrating paracentesis of the big bubble was performed centrally with a consecutive bubble prolapse. A fine spatula was introduced to the space between the DM and the remaining stroma. A size 15 blade knife was used to cut the tissue above the spatula. The cornea was cut into 4 quadrants. Corneal scissors (Castroviejo) were used to remove the quadrant in order to leave a bare descement, upon removal of the fourth quadrant a DM perforation was observed. Therefore the procedure was converted to PKP. The $7.75 \mathrm{~mm}$ donor cornea button was sutured to the recipient by 16 partial thickness 10-0 nylon interrupted sutures.

The first postoperative follow-up took place 1 day following the surgery. The patient's visual acuity was 3 meter finger count. Upon slit lamp examination there was what appeared to be a remnant of a loose folded DM attached to the surgical wound edge forming a 'double anterior chamber'. At that point the 'watch and wait' approach was chosen. Topical neomycin sulfate combined with dexamethasone sodium phosphate had been prescribed for the patient.

Visual acuity was $6 / 24$ one month following the surgery. The corneal graft was clear while the DM remnant persisted. Taking into account the improvement in visual acuity and lack of visual complaints on the part of the patient, removing the DM was not considered.

Five months following surgery, the visual acuity had declined to $2 \mathrm{~m}$ finger count. Upon slit lamp examination the DM appeared to be tighter and opaque obscuring the visual axis. An imaging of the anterior chamber performed using Pentacam confirmed the clinical impression (Fig. 1). Corneal topography demonstrated astigmatism with nearly 10 diopter cylinder at 60 a degree axis (Fig. 2). The patient continued using topical dexamethasone and artificial tears. 


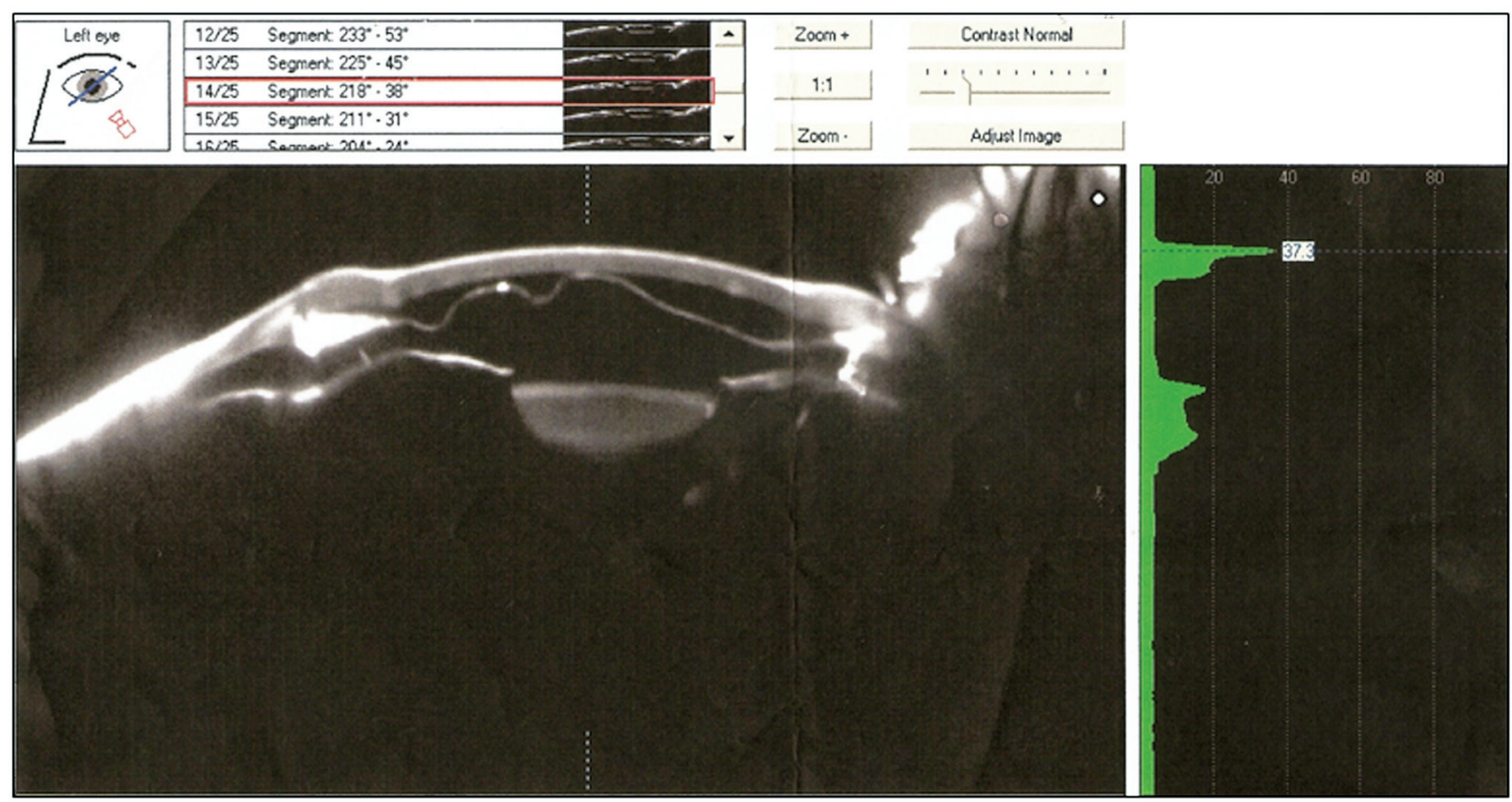

Fig. 1: Pentacam image demonstrating a loose DM dividing the anterior chamber

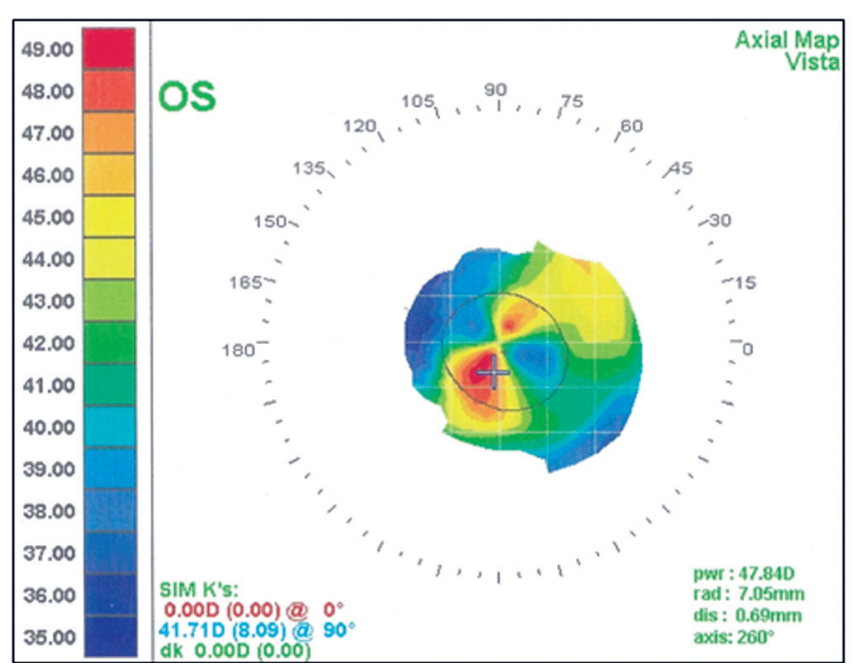

Fig. 2: Corneal topography demonstrating a 10 diopter cylinder at $60^{\circ}$

By the 9 months following surgery the visual acuity was $6 / 60$ improving to $6 / 36$ using pinhole. Upon examination the DM appeared to be opaque, thicker and tighter (Fig. 3). This finding was confirmed as well by the imaging of the anterior chamber (Fig. 4). An attempt to create an opening through the thickened DM using YAG laser technique had failed most probably owing to its rigid texture and thickness.

Eventually, the central portion of the membrane was excised using a stiletto knife entering the anterior chamber though paracentesis performed in the space between the corneal graft and the DM. Finally, the excised membrane

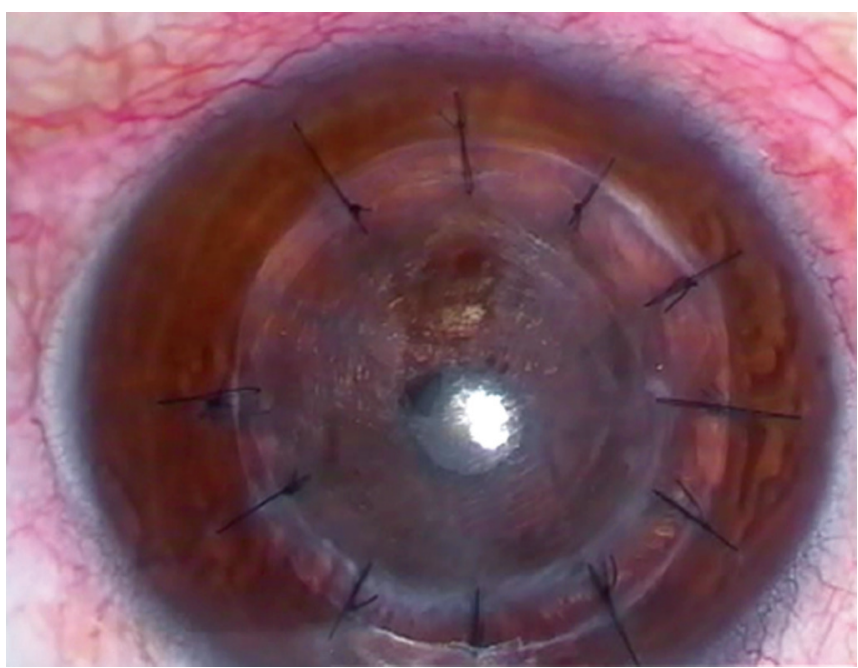

Fig. 3: The left eye showing an opaque DM underlying a clear graft

was removed through a $3.2 \mathrm{~mm}$ surgical opening performed in the peripheral host cornea.

A year following the second surgery the visual acuity was $6 / 18$. A central opening of the DM was apparent upon examination (Fig. 5) and imaging using Pentacam (Fig. 6). Corneal topography demonstrated astigmatism of an 18 diopter cylinder at $150^{\circ}$ axis (Fig. 7).

A corneal graft endothelial failure and cataract formation was documented 2 years following the second surgery. The patient underwent a second corneal transplant and cataract surgery with insertion of a secondary intraocular lens into the posterior chamber. 

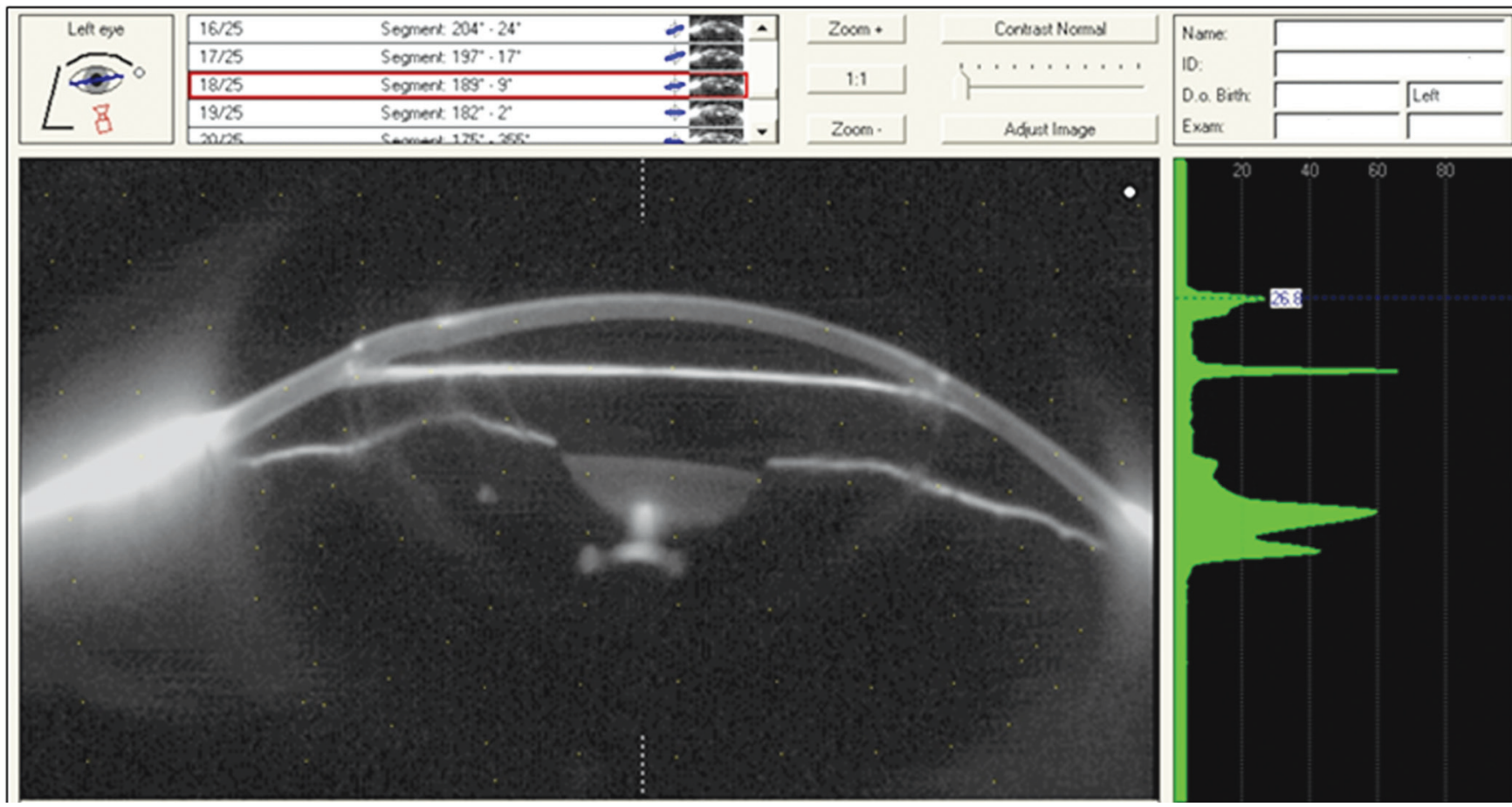

Fig. 4: Pentacam image demonstrating a tight and thick DM

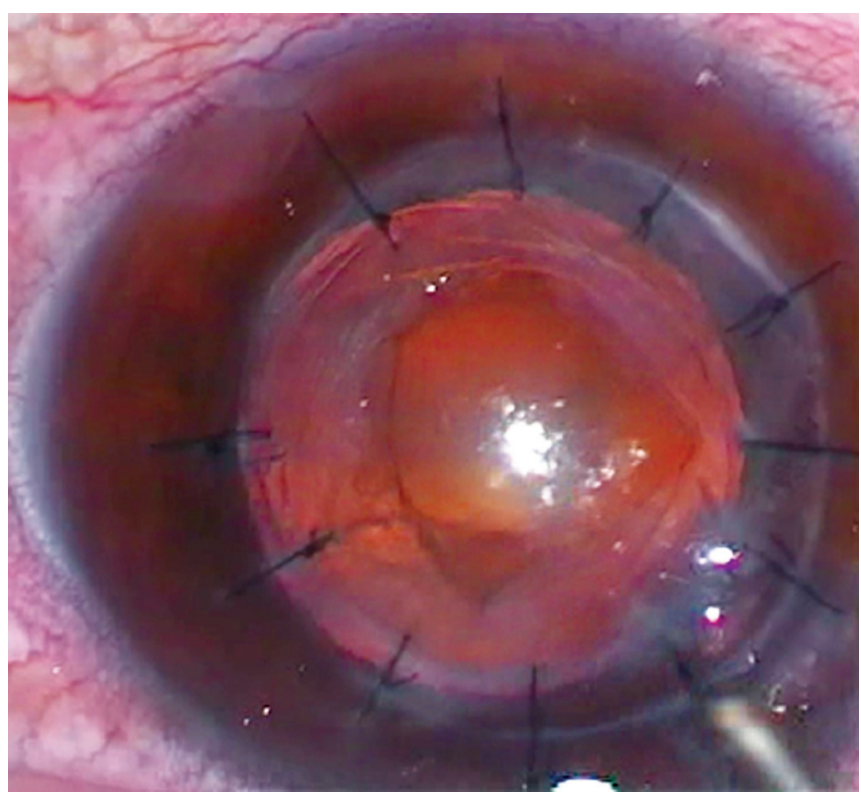

Fig. 5: The left eye following the excision showing a central opening through the opaque membrane

\section{DISCUSSION}

In this case report a patient underwent a DALK operation that was converted into PKP. While in a PKP the host's DM is normally removed completely at the area underlying the graft, in the above case the DM was unintentionally removed partially. A possible explanation for the DM remnant's presence could be due to its having been scrolled outside of the surgeon's view (Fig. 8). On the first postoperative day the membrane appeared unfolded, tending toward its natural location, thereby forming a double anterior chamber. While in a DALK operation a possible complication is DM detachment, one would not expect the presence of a membrane following PKP. In the former condition endothelial pumping activity and its deturgescent effect could lead to spontaneous reattachment of the DM within a few months. ${ }^{6,7}$ The latter condition apparently necessitates early intervention such as gas injection into the anterior chamber forcing the loose DM toward the posterior corneal graft. Another possible solution would be YAG laser to deflect the undesirable membrane away from the visual axis; the success of such a method would depend upon the tissue's properties, for example thickness and rigidity. In this case, withholding treatment eventually led to a stiffening of the membrane which could not be affected by laser. The thickening of the membrane could stiffen and distort the globe, causing significant astigmatism as demonstrated in the corneal topography above. Finally, a surgical excision of the membrane was performed. Such manipulation inside the anterior chamber may have accelerated the process of graft failure. Therefore, early intervention might have been preferable. 


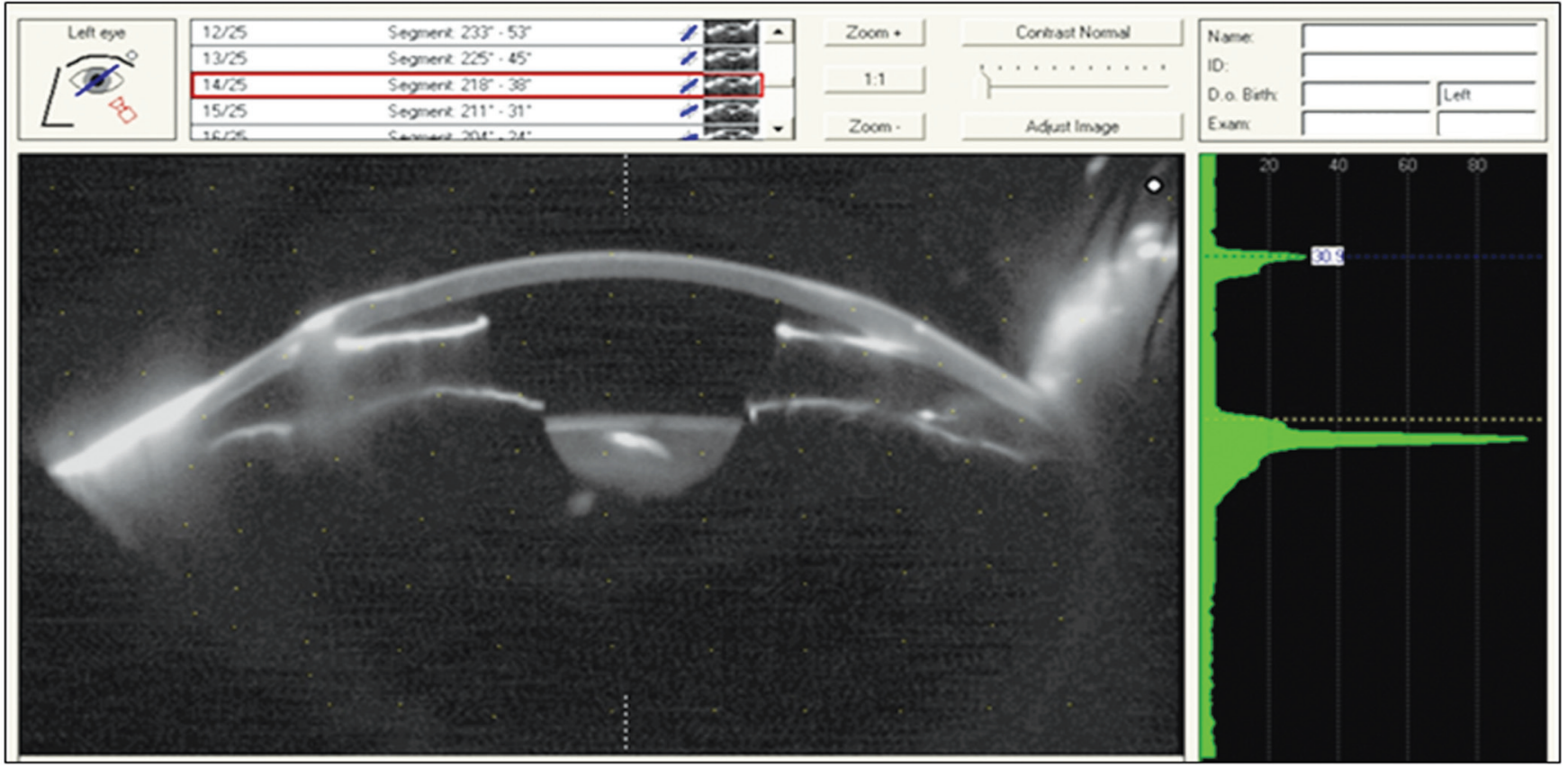

Fig. 6: Pentakam image following the excision demonstrating the remaining DM at the peripheral anterior chamber

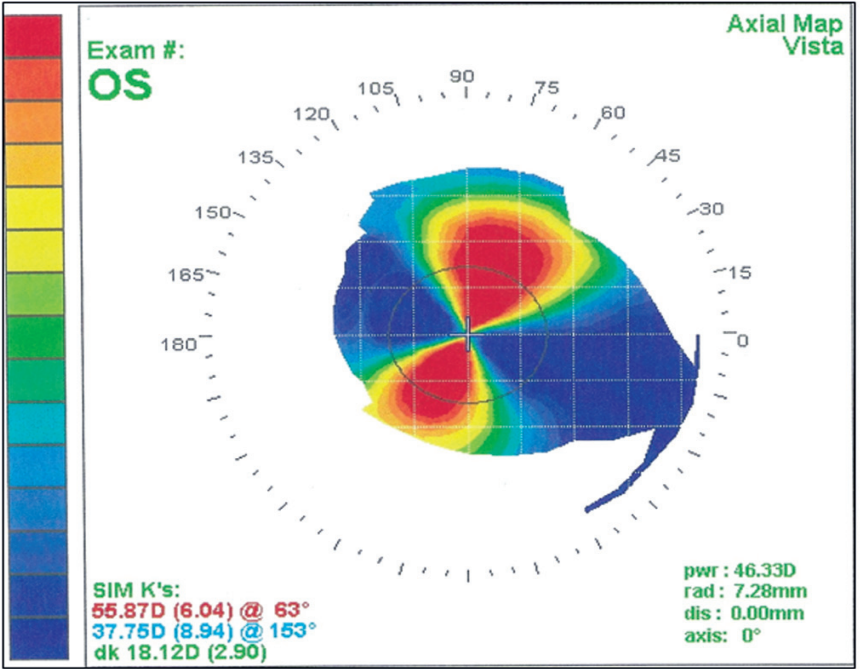

Fig. 7: Corneal topography following the second surgery demonstrating an 18 diopter cylinder at $150^{\circ}$

\section{REFERENCES}

1. Mohammed A, Teichmann KD. Big-bubble technique to bare Descemet's membrane in anterior lamellar keratoplasty. J Cataract \& Refractive Surgery 2002;28(3):398-403.

2. Kubaloglu A, et al. Long-term results of deep anterior lamellar keratoplasty for the treatment of keratoconus. Am J Ophthalmol 2011;151(5):760-767.

3. Juntaro S, Kondo J. Deep lamellar keratoplasty with complete removal of pathological stroma for vision improvement. British J Ophthalmol 1997;81(3):184-188.

4. Lin TK, Ibrahim M, Kaye SB. Spontaneous resolution of Descemet membrane detachment after deep anterior lamellar keratoplasty. Cornea 2006;25(1):104-106.

5. Yakov G, Zadok D, Avni I. Spontaneous resolution of Descemet membrane detachment following big-bubble deep anterior lamellar keratoplasty. Euro J Ophthalmol 2009;19(6):1079-1081.

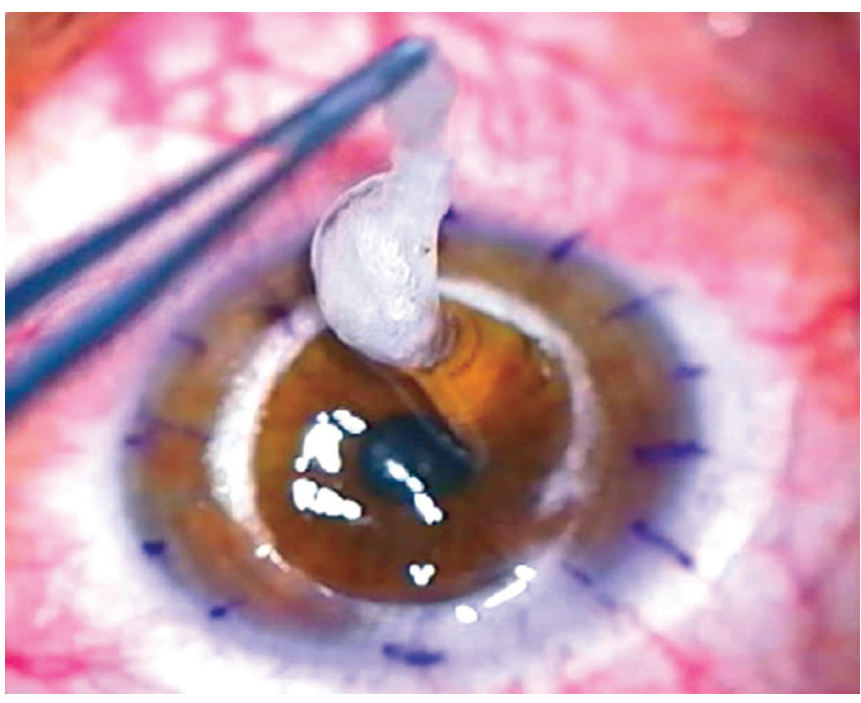

Fig. 8: Photo taken during surgery showing what later proved to be the process of partial removal of the DM

6. Waring GO 3rd, et al. The corneal endothelium. Normal and pathologic structure and function. Ophthalmology 1982; 89(6):531-590.

7. John HE. The physiologic control of corneal hydration. Am J Ophthalmol Part 2 1957;44(5):262.

\section{ABOUT THE AUTHORS}

\section{Shmuel Grafi (Corresponding Author)}

Department of Ophthalmology, Faculty of Medicine, Bruch Padeh Medical Center, Bar Ilan University, Poriya 15208, Israel, Phone: +972547956551, e-mail: sgraffi@walla.com

\section{Modi Naftali}

Department of Ophthalmology, Faculty of Medicine, Bruch Padeh Medical Center, Bar Ilan University, Poriya, Israel 